\title{
Quantitative anterior segment optical coherence tomography
}

\section{Tomografía de coherencia óptica cuantitativa del segmento anterior}

\author{
Sergio $\operatorname{Ortiz}^{(*, S)}$, Susana $\operatorname{Marcos}(\mathrm{S})$ \\ Instituto de Óptica “Daza de Valdés”, Consejo Superior de Investigaciones Científicas, C/Serrano 121, \\ 28006, Madrid, Spain. \\ (*) Email: $\underline{\text { sortiz@io.cfmac.csic.es }}$ \\ S: miembro de SEDOPTICA / SEDOPTICA member \\ Received / Recibido: 02/07/2014. Revised / Revisado: 19/08/2014. Accepted / Aceptado: 20/08/2014.
}

DOI: http://dx.doi.org/10.7149/OPA.47.3.227

\begin{abstract}
:
We present in this paper the quantification of the geometrical features of the anterior segment of the eye using Anterior Segment Optical Coherence Tomography (AS-OCT). This quantification was achieved by following our own developed methodology including the device development and their distortion calibration (Fan and refraction) for 3-D imaging, and automatic analysis tools as well. The stastistical comparison of the corrected topographies of the anterior cornea with topographies from state-of-the art clinical topography systems represents a valuable contribution toward the development "All-OCT-based" topographic systems. In addition, we first reported topographies of the crystalline lens in vivo, which is a promising tool to investigate the lens optical properties, the contribution of the lens to the overall retinal image quality, and understanding the change of the physical properties of the lens with accommodation and aging. Furthermore, our quantitative ASOCT allowed the analysis of the keratoconic cornea and the changes produced by Intra Corneal Ring Segment (ICRS) treatment, as well as monitoring of the ICRS 3-D location. Finally, OCT-based biometry we present in this paper is a promising tool to investigate the changes after cataract surgery; the contribution of the (Intra Ocular Lens) IOL location and tilt to the overall retinal image quality, and in the design of new IOLs.
\end{abstract}

Key words: Optical Coherence Tomography, Surface Measurements, Optical Instruments, ThreeDimensional Image Acquisition, Visual Optics, Ophthalmic Instrumentation.

\section{RESUMEN:}

Presentamos en este artículo la cuantificación de los aspectos geométricos del segmento anterior del ojo empleando Tomografía de Coherencia Óptica del segmento anterior (AS-OCT). Esta cuantificación se logró mediante el desarrollo de nuestra metodología que incluye el desarrollo de dispositivos y la calibración de sus distorsiones (Fan y refracción) para imagen 3-D, así como el desarrollo de herramientas de procesado automáticas. La comparación estadística de las topografías corregidas de la superficie anterior de la córnea comparada con aquellos topógrafos estándares en el ámbito clínico, supone una contribución hacia los sistemas topográficos basados únicamente en OCT. Además, reportamos las primeras topografías del cristalino in vivo, lo que convierte al OCT en una herramienta prometedora en el estudio de las propiedades ópticas del cristalino, su contribución en la calidad de imagen retiniana, así como comprender los cambios de sus propiedades físicas con la acomodación y la edad. Nuestro AS-OCT cuantitativo permitió el análisis de corneas con queratocono, así como analizar los cambios producidos por el tratamiento con ICRS y su localización 3-D. Por último, la biometría basada en OCT, que presentamos en este artículo, es una herramienta prometedora para investigar los cambios después de cirugía de cataratas; la contribución del emplazamiento de la IOL y de su inclinación en la calidad de la imagen retiniana y en el nuevo diseño de nuevas IOL.

Palabras clave: Tomografía de Coherencia Óptica, Medida de Superficies, Instrumentos Ópticos, Adquisición de Imagen Tridimensional, Óptica Visual e Instrumentación Oftalmológica. 


\section{REFERENCES AND LINKS / REFERENCIAS Y ENLACES}

[1]. D. Huang, E. A. Swanson, C. P. Lin, J. S. Schuman, W. G. Stinson, W. Chang, M. R. Hee, T. Flotte, "Optical coherence tomography", Science 254,1178-1181 (1991). DOI

[2]. J. A. Izatt, M. R. Hee, E. A. Swanson, "Micrometer scale resolution imaging of the anterior eye in vivo with optical coherence tomography", Arch. Ophthalmol. 112, 1584-1589 (1994). DOI

[3]. Y. Li, R. Shekhar, D. Huang, "Corneal pachymetry mapping with high-speed optical coherence tomography", Ophthalmology 113, $792-799$ (2006). DOI

[4]. M. Avila, Y. Li, J. C. Song, D. Hang, "High-speed optical coherence tomography for post-LASIK management", J. Cataract Refrac. Surg. 32, 1836-1842 (2006). DOI

[5]. M. M. Lai, M. Tang, E. M. Andrade, Y. Li, R. N. Khurana, J. C. Song, D. Huang, "Optical coherence tomography to assess intrastromal corneal ring segment depth in keratoconic eyes", J. Cataract Refract. Surg. 32, 1860-1865 (2006). DOI

[6]. Y. Li, D. M. Meisler, M. Tang, A. T. H. Lu, V. Thakrar, B. J. Reiser, D. Huang, "Keratoconus diagnosis with optical coherence tomography pachymetry mapping", Ophthalmology 115, 2159-2166 (2008). DOI

[7]. G. Savini, E. Goto, M. Carbonelli, P. Barboni, D. Huang, "Agreement between Stratus and Visante optical coherence tomography systems in tear meniscus measurements", Cornea 28, 148-151 (2009). DOI

[8]. S. Radhakrishnan, J. Goldsmith, D. Huang, V. Westphal, D. K. Dueker, A. M. Rollins, J. A. Izatt, S. D. Smith, "Comparison of optical coherence tomography and ultrasound biomicroscopy for detection of narrow anterior chamber angles", Archive of Ophthalmology 123, 1053-1059 (2005a). DOI

[9]. R. N. Khurana, Y. Li, M. Tang, M. M. Lai, D. Huang, "High-speed optical coherence tomography of corneal opacities", Ophthalmology $114,1278-1285$ (2007). DOI

[10].J. A. Goldsmith, Y. Li, M. R. Chalita, V. Westphal, C. A. Patil, A. M. Rollins, J. A. Izatt, D. Huang, "Anterior chamber width measurement by high-speed optical coherence tomography", Ophthalmology 112, 238-244 (2005). DOI

[11]. M. Zhao, A. N Kuo, J. A. Izatt, "3D refraction correction and extraction of clinical parameters from spectral domain optical coherence tomography of the cornea", Opt. Express 18, 8923-8936 (2010). DOI

[12]. M. Gora, K. Karnowski, M. Szkulmowski, B. J. Kaluzny, R. Huber, A. Kowalczyk, M. Wojtkowski, “Ultra high-speed swept source OCT imaging of the anterior segment of human eye at $200 \mathrm{kHz}$ with adjustable imaging range", Opt. Express 17, 14880-14894 (2009). DOI

[13]. K. Karnowski, B. J. Kaluzny, M. Szkulmowski, M. Gora, M.Wojtkowski, "Corneal topography with highspeed swept source OCT in clinical examination", Biomed. Opt. Express 2, 2709-2720 (2011). DOI

[14]. S. Ortiz, D. Siedlecki, L. Remon, S. Marcos, "Optical coherence tomography for quantitative surface topography", Appl. Opt. 48, 6708-6715 (2009). DOI

[15]. S. Ortiz, D. Siedlecki, I. Grulkowski, L. Remon, D. Pascual, M. Wojtkowski, S. Marcos, "Optical distortion correction in optical coherence tomography for quantitative ocular anterior segment by threedimensional imaging", Opt. Express 18, 2782-2796 (2010). DOI

[16]. I. Grulkowski, M. Gora, I. Szkulmowski, I. Gorczynska, D. Szlag, S. Marcos, A. Kowalczyk, M. Wojtkowski, "Anterior segment imaging with spectral OCT system using a high-speed CMOS camera," Opt. Express 17, 4842-4858 (2009). DOI

[17].S. Ortiz, D. Siedlecki, P. Pérez-Merino, N. Chia, A. de Castro, M. Szkulmowski, M. Wojtkowski, S. Marcos, "Corneal topography from spectral optical coherence tomography (sOCT)", Biomed. Opt. Express 2, 3232-3247 (2011). DOI

[18].S. Ortiz, P. Pérez-Merino, N. Alejandre, E. Gambra, I. Jimenez-Alfaro, S. Marcos, "Quantitative OCTbased corneal topography in keratoconus with intracorneal ring segments", Biomed. Opt. Express 3, 814-824 (2012). DOI

[19].S. Ortiz, P. Pérez-Merino, E. Gambra, A. de Castro, S. Marcos, "In vivo human crystalline lens topography", Biomed. Opt. Express 3, 2471-2488 (2012). DOI 
[20]. S. Ortiz, P. Pérez-Merino, S. Durán, M. Velasco-Ocana, J. Birkenfeld, A. de Castro, I. Jiménez-Alfaro, S. Marcos, "Full OCT anterior segment biometry: an application in cataract surgery", Biomed. Opt. Express 4, 387-396 (2013). DOI

[21].S.Ortiz, D. Siedlecki, L. Remon, S. Marcos, "Three-dimensional ray tracing on Delaunay-based reconstructed surfaces", Appl. Opt. 48, 3886-3893 (2009). DOI

[22]. S. R. Uhlhorn, D. Borja, F. Manns, J. M. Parel, "Refractive index measurement of the isolated crystalline lens using optical coherence tomography", Vision Res. 48, 2732-2738 (2008). DOI

[23]. A. de Castro, P. Rosales, S. Marcos, "Tilt and decentration of intraocular lenses in vivo from Purkinje and Scheimpflug imaging validation study", J. Cataract Refract. Surg. 33, 418-429 (2006). DOI

[24]. P. Rosales, M. Wendt, S. Marcos, A. Glasser, "Changes in crystalline lens radii of curvature and lens tilt and decentration during dynamic accommodation in rhesus monkeys", J. Vision 8, 1-12 (2008). DOI

[25]. M. Dubbelman, V. A. Sicam, G. L. van der Heijde, "The shape of the anterior and posterior surface of the aging human cornea", Vision Res. 46, 993-1001 (2006). DOI

[26]. D. Borja, D. Siedlecki, A. de Castro, S. Uhlhorn, S. Ortiz, E. Arrieta, J. M. Parel, S. Marcos, F. Manns, "Distortions of the posterior surface in optical coherence tomography images of the isolated crystalline lens: effect of the lens index gradient", Biomed. Opt. Express 1, 1331-1340 (2010). DOI

[27]. A. Gullstrand, Helmholtz's Physiological Optics, Optical Society of America, New York, Apendix, pp. 350-358 (1924).

[28]. H. Liou, N. A. Brennan, "Anatomically accurate, finite model eye for optical modelling", J. Opt. Soc. Am. $A$ 14, 1684-1695 (1997). DOI

[29]. M. Dubbelman, G. L. van der Heijde, "The shape of the aging human lens: curvature, equivalent refractive index and the lens paradox", Vision Res. 41, 1867-1877 (2001). DOI

\section{Introduction}

The optical quality of the human eye is mainly determined by the geometrical and optical properties of two elements, cornea and crystalline lens. The cornea accounts for twothirds of the optical refractive power, while the crystalline lens provides approximately one third of the total static refractive power of the eye, and it is the responsible for accommodation. Accurate description of the geometry of the eye's optical components is particularly critical for understanding their contribution to optical aberrations. Quantitative biometry and geometrical anterior segment features are essential in many areas in physiological optics (i.e. customized eye modeling understanding of the structural changes in the crystalline lens during accommodation and aging, crystalline lens gradient index of refraction reconstruction, etc...) and ophthalmology (i.e. changes in the anterior and posterior corneal topography after refractive surgery or after Intra corneal Rings segments for keratonus treatment) and the accuracy and evaluation of cataract surgery.

Anterior segment characterization is limited only to various commercial instruments, which provide images of anterior and posterior corneal surfaces and quantitative geometrical information of the cornea. However, there are not commercially available instruments that provide crystalline lens information. Scheimpflug imaging, which allows acquisition of cross-sectional images of the cornea and lens with the proper correction and validation it is able to provide quantitative geometrical features and topographic maps of the cornea as well as, radius of curvature, and asphericity of the lens surfaces. The shape, location and phakometry of the crystalline lens have been reported in vivo and in vitro using different imaging techniques. Radii of curvature, tilt and decentration of the lens have been measured using a Purkinjeimaging based method. In addition to optical techniques, the anterior chamber of the eye can be imaged through non-optical imaging techniques such as ultrasound and Magnetic Resonance Imaging. These non-optical techniques allow visualization of the entire crystalline lens and its neighboring ocular structures. However, these are either invasive or time-consuming imaging methods, with significantly lower resolution than optical techniques, which impose major problems (low acquisition speed, motion artifacts or low 
sampling density), which prevent quantifying the crystalline lens geometry with high accuracy

Optical Coherence Tomography (OCT) is one of the most versatile and promising imaging techniques of the anterior segment of the eye, due to its high resolution, and the possibility of imaging the anterior and posterior cornea, the iris and the crystalline lens over a large crosssectional area. It was developed in 1991 [1], as a non-invasive technique for characterizing surfaces and faint tissue. Since then, the technique has experienced an increasing interest by engineering, research and clinical communities. In that seminal paper, the authors demonstrated the first in vitro OCT images of the human retina and coronary arterial wall, suggesting the potential of OCT as a new inspection tool for imaging quasi-transparent or scattered media. The Anterior Segment OCT or (AS-OCT) was first reported in 1994 when the first Real-time OCT, in a time-domain configuration, for anterior segment was developed at Duke University [2]. Since then, numerous Clinical applications of AS-OCT instruments have been reported. These include: pachymetric measurements of flap and stroma in LASIK surgery [3], Post-refractive surgery corneal evaluation [4], in Intra Corneal Rings Surgery (ICRS) [5], of Keratoconus diagnosis [6], Tear meniscus evaluation [7], for Glaucoma diagnosis by angle measurements for narrowangle [8], visualization of corneal scars [9], biometric measurements for IOL power calculations [10]. There have also been several attempts of quantifying parameters of the ocular optical components, for instance Izatt's group [11] extracted the keratometric parameters of the cornea and they compared them with those obtained from other ophthalmic devices. The cornea was scanned meridionally, and only corneal power, rather than corneal topography was reported. Gora et al [12] presented a preliminary quantitative corneal analysis based on a swept source OCT using a meridian rastering protocol for collecting data. They presented a volumetric and real time reconstruction of dynamic processes, such as pupillary reaction to light stimulus or blinkinduced contact lens movements. [13] presented a corneal topography In a keratoconic subject in comparison with corneal topographies obtained from clinical instruments: videokeratographer, Scheimpflug camera and slit scanning system. Although compensation of fan distortion in this data set was not reported. However, 3-D OCT quantitative geometrical parameters can only be retrieved accurately upon correction of the fan (scanning) and optical (refraction) distortion $[14,15]$.

The developed technique has been applied to the geometrical characterization of the cornea and the lens, in normal, pathological and treated eyes. The normal eyes were used to obtain anterior topographic maps comparable to standard devices in clinics (Placido Ring videokeratography and Scheimpflug camera), and for providing for the first time, to our knowledge, 3-D elevation maps of the anterior and posterior lens surfaces of the eye in vivo. We also present the quantification of the keratoconic cornea before and after ICRS (Intra Corneal Ring Segment) implantation. To our knowledge, this is the first time where fully quantitative OCT has been applied to retrieve anterior and posterior corneal elevations and pachymetric to irregular and treated corneas. Finally, we provide the biometry of a cataract patient before and after implantation of an IOL (Intra Ocular Lens). To our knowledge, this is the first report of 3-D lens alignment measurements with OCT, and of the full in vivo anterior segment quantification for a patient before and after cataract surgery.

\section{Methods}

\section{2.a. Laboratory-based spectral OCT system}

The analysis was performed on images collected from a custom-developed spectral OCT system (from collaborative effort with Copernicus University, Torun, Poland) [16]. The set-up is based on a fiber-optics Michelson interferometer configuration with a superluminescent diode SLD $\left(\lambda_{0}=840 \mathrm{~nm}, \Delta \lambda=50 \mathrm{~nm}\right.$; Superlum, Ireland) as a light source and a spectrometer consisting of a volume diffraction grating and a 12-bit line-scan CMOS camera with 4096 pixels (Basler sprint spL4096-140k; Basler AG, Germany) as a detector. The effective acquisition speed is 25000 A-Scans/s, which optimized balance between speed and SNR. The axial range 
of the instrument is $7 \mathrm{~mm}$ in depth, resulting in a theoretical pixel resolution of $3.4 \mu \mathrm{m}$. The axial resolution predicted by the bandwidth of the superluminescent diode laser source is $6.9 \mu \mathrm{m}$.

\section{2.b. Experiments in vivo}

In this paper it is presented different in vivo experiments. Topographic maps of normal subjects from anterior cornea and lens, and it is also presented the characterization of two pathologies before and after the surgery. The characterization of the keratoconus before and after the implantation of ICRS and a patient implanted before and after the cataract surgery. For all of this experiments the SLD power exposure was fixed at $800 \mu \mathrm{W}$. Focus was changed by an automatic displacement system to achieve optimal imaging of the different anterior segment structures (cornea, anterior and posterior lens/IOL). In order to minimize the impact of motion artifacts, the image acquisition time was set to $0.72 \mathrm{~s}$. The subjects were aligned to the AS-OCT system by using the specular reflection of the cornea as a reference. All studies were approved by Institutional Review Boards and followed the tenets of the Declaration of Helsinki. The subjects signed a consent form after receiving an explanation of the nature of the study. All the measurements were using a common protocol using $50 \mathrm{~B}$-Scans composed by a collection of 360 . For the corneal experiments the zone was $10 \times 12,10 \times 15$ for the lens and $7 \times 15$ for the Biometry study.

Anterior corneal topography: A total of 10 eyes from five human subjects participated in the experiment. The ages ranged from 24 to 38 years. The subjects were considered normal in a clinical ophthalmological examination. In addition to the AS-OCT measurements, corneal topographies were obtained from commercial Scheimpflug topography (Pentacam, Oculus Optikgeräte GmbH, Wetzlar, Germany) and Placido based videokeratography (instrument of section 2.4). These instruments were used for comparison with state-of-the art clinical standards, not as gold-standard references. The 50 -scan acquisition protocol was used in the Pentacam instrument. Each Pentacam measurement was collected in about 2 seconds, while the subject fixated foveally the built-in red fixation spot, and the corneal reflex of the fixation spot used as a reference. Each Placido based videokeratography measurement was collected in about $300 \mathrm{~ms}$. Alignment was achieved by centering the image of the reflected rings on the cornea with the corneal reflex. Five repeated sets of data were obtained per subject with each instrument.

Lens Topography: Images were collected on the right eye of 3 young subjects (ages 28-33). Measurements were performed under mydriasis (by Tropicamide 1\%). The subjects were stabilized using a bite bar. Alignment of the subject was achieved with respect to the anterior corneal specular reflection, while the subject fixated on a reference maltese cross target projected on a minidisplay at optical infinity. A total of 15 sets of 3-D data were collected in each eye: 5 repeated images of the cornea, 5 repeated images of the anterior part the lens, and 5 repeated images of the posterior part of the lens.

Keratoconus ICRS implantation: Measurements on the patient were performed pre-operatively and 30 days after ICRS implantation. The patient was stabilized using a bite bar. Alignment of the patient was achieved with respect to the anterior corneal specular reflection. All the measurements were performed in a dark room under the same conditions without the use of dilating drops. A total of five sets of 3-dimensional measurements were collected pre-operatively and post-ICRS implantation.

Biometry study: Images were collected on the left eye of a 73 year-old cataract patient before (15 days) and after (90 days) IOL implantation. Measurements were performed under natural conditions pre-operatively and under mydriasis (phenylephrine $10 \%$ ) post-operatively. The subject was stabilized using a bite bar. Alignment of the subject was achieved with respect to the anterior corneal specular reflection, while the subject fixated on a reference E-letter target projected on a minidisplay at optical infinity. A total of 15 sets of 3-D data were collected pre-operatively (3 repeated images for the cornea, anterior lens and posterior lens, respectively), and 9 sets post- 
operatively (3 repeated images for the cornea and IOL, respectively). All 3-D sets of data contained the iris volume (fixed reference for merging).

\section{2.c. 3-D Image processing tool}

The image processing algorithm can be summarized in 10 steps. The first 9 steps have been described with a more degree of detail in previous studies [14,15,17-20].

(1) Denoising: A rotational kernel transform was performed for edge-preserving denoising, using a mask of size 9 pixels. In addition, a wavelet low-pass filtering processing based on log-Gabor wavelet for 7 scales and 6 orientations was used. (2) Statistical thresholding: An adaptive algorithm based on a multimodal Gaussian fitting of the histogram intensity was performed on the entire 3-D sets of data, which allowed the separation of a noise class from the signal. (3) As a result of the overlapping statistics of the noise and signal, randomly distributed noise is still present in the images after thresholding. We developed a volume clustering algorithm based on area or volume classification. Volumes of connected points are identified as classes. The classes with a volume size below a certain threshold are eliminated. (4) Multilayer segmentation: Automatic segmentation was based on Canny detection in each A-scan (1-D signals). In an initial step, a Gaussian filter (standard deviation, 5 pixels) was convolved with the signal to reduce spurious peaks associated to noise detection in the CMOS line-camera. The first derivatives of the signal were then obtained by convolving the resultant A-Scan with finite-size linear-phase separable kernels for differentiation of the discrete data (A-Scan). The size of the kernels (3-9 pixels) controls the sensitivity of the algorithm to noise (the lower the number of pixels used for the kernel the higher the noise sensitivity). The result of the first derivative calculation provides both local maxima and minima of the A-scan. Maxima are identified by subtraction of the slope sign of two consecutive points (positive values). (5) Pupil center reference: The pupil center was used as a reference across measurements (i.e. cornea, anterior and posterior lens) and to define the optical zone (effective area within the pupil). The pupil center shifts little across pupil sizes and light conditions. The 3-D position of the pupil center (including the axial coordinate), and estimation of the pupil plane in 3-D, as this information is used for registration and merging of the corneal and lens images. The lateral pupil coordinates were obtained by fitting the inner iris zone to an ellipse, while the axial coordinate was obtained by non-linear least-square fitting of the iris plane in 3-D (and evaluation of the plane at the estimated pupil lateral coordinates). In addition, the characteristic vector of the iris plane was obtained, which provided the tilt angle of this plane with respect to the OCT coordinate system. (6) 3-D volume merging: The pupil coordinates are assumed as a common reference for the images of the anterior segment collected at different depths. Variations in the pupil diameter were negligible across images. Images of the cornea, anterior and posterior lens were merged using the pupil center and pupil plane orientation for registration. In a first step prior to merging, the corneal image was inverted, as for efficiency in the focus range shift, the cornea was acquired in the opposite side of the Fourier transform (in comparison with the crystalline lens acquisition). The 3-D volumes of the anterior cornea/iris and posterior lens/iris are shifted to the pupil center reference, and rotated (using a rotation matrix in order to superimpose the characteristic vectors of the corresponding pupil plane to those of the anterior lens/iris. (7) Geometrical distances calculation and further denoising: The registration of the 3-D volumetric data sets involved shift, rotation and resampling using a Delaunay surface description for interpolation [21]. Optical distances were calculated by direct subtraction of the coordinates of the different surfaces, and geometrical distances were obtained by dividing the optical distance by the corresponding group refractive index 1.376 for the cornea, 1.336 for the aqueous humor, agedependant homogeneous refractive index for the crystalline lens of the subject in the study as per a previously published age-related expression [22], 1.430 for the Crystalens IOL, 1.486 for the PMMA ICRS). (8) Distortion corrections: In order to correct the interface layers for the effect of distortion in general (fan distortion and 
refraction), it is required to propagate the geometrical distances obtained from the previous point along the directional cosines of the ray, taking into account the refraction that occurs at each interface between two media of different refractive indices. A procedure similarly to that described in an earlier publication and practically applied to the cornea was followed, expanding it sequentially from the cornea to the posterior surface of the crystalline lens. Correction of the fan and optical distortions produces large geometrical changes in the anterior optical surfaces, which are qualitatively observable in the diameter of the visible areas of the anterior, and to a larger extent, of the posterior lens. (9) Surface fitting by quadrics (biconicoid, conicoid), and elevation maps: The surfaces were fitted by quadrics in a 5-6 diameter optical zone depending on the experiment with respect to their apexes. The elevation maps were represented as the difference of elevation data from the reference sphere. Thickness maps of the lens were obtained by direct subtraction of the anterior elevation map from the posterior. All maps weredisplayed in a square grid of $100 \times 100$

points respect to the pupil center. The maps were represented using the so called "jet" color map in Matlab notation, where warm colors depict high differences while cold colors depict low differences. (10) Anterior segment biometry: central corneal thickness (CCT), anterior chamber depth (ACD), crystalline lens thickness (CLT), intraocular lens thickness (ILT), intraocular lens position (ILP), and lens IOL tilt (IT) were computed from the corrected 3-D OCT images. CCT is defined as the distance between the anterior and posterior corneal apexes. ACD is defined as the distance between the posterior corneal surface apex and the anterior crystalline lens surface apex. CLT and ILT are defined as the distance between the anterior and posterior lens surface apexes. ILP is defined as the distance between the posterior corneal surface apex and the anterior IOL surface apex. The 3-D Euclidean distances were obtained by direct subtraction of
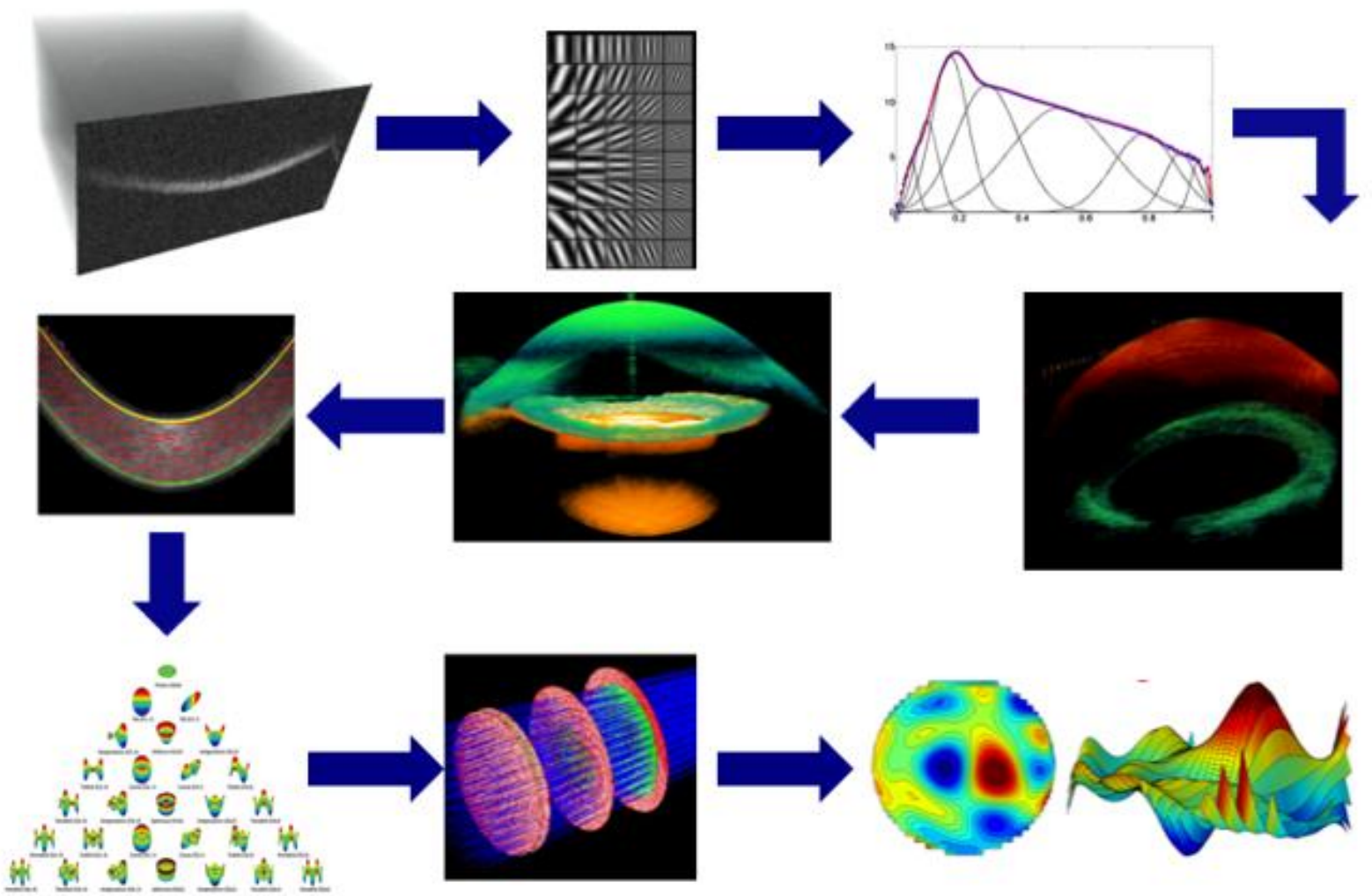

Fig. 1. Illustration of some image processing steps, from top to bottom and from left to right: Input data, denoising, Statistical thresholding, volume clustering, merging, multilayer segmentation, surface denoising (Zernike expansion), Fan and refraction correction and output data. 


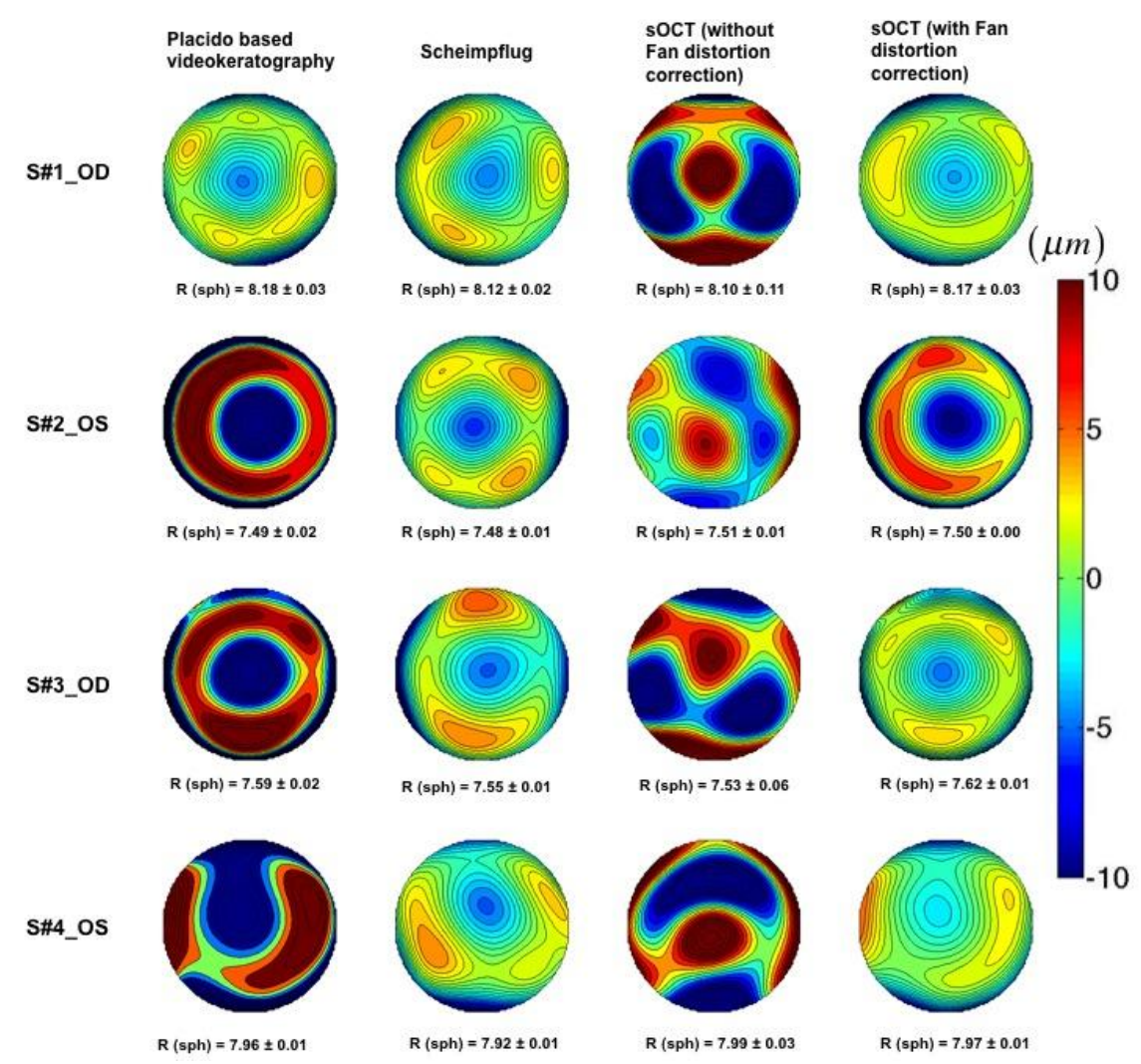

Fig. 2. Corneal elevation maps obtained in 4 eyes obtained from different instruments (relative to the best fitting sphere). $\mathrm{R}=$ radii of curvature of the best fitting sphere (from fits to sphere quadrics).

the apexes coordinates obtained from the fittings of the surfaces to spheres, after optical distortion correction. Crystalline lens/IOL decentration is defined as the lateral Euclidean distance between the IOL center and the pupil center. Lens tilt is defined as the angle between the axis of the lens and the pupillary axis. The Lens/IOL axis (L) is defined as the vector that joins the apexes of the anterior and posterior lens surfaces [23]. The pupillary axis (P) is defined as the vector that joins the center of curvature of the anterior cornea and the pupil center. The angle between axes is obtained by the scalar product of those vectors as it is explained in reference [23]. The horizontal and vertical components of the tilt are obtained, following a sign convention similar to that reported by Rosales et al [24].

\section{Results}

We are going to present the application of the developed methodology in vivo patients. This section includes the anterior corneal topographic maps of 4 subjects, the first topographies of the human crystalline lens (corrected from distortions) in 3 subjects, the characterization of the ICRS surgery in a patient and the biometric changes experimented by a cataract patient after the implantation of an IOL.

\section{3.a. Anterior Corneal topography}

Figure 2 shows topographic height maps from 4 eyes, obtained from Placido-based videokeratography, Scheimpflug and AS-OCT (without and with fan distortion correction). Difference maps are not shown, as none of the instruments is considered as a "gold standard". All maps are presented on the same scale for each eye, and are averages of 5 repeated measurements. All data are represented over a 6-mm diameter zone, centered at the specular reflection for foveal fixation. The topographic maps were obtained by fitting the raw elevation maps to a 10th degree Zernike expansion, relative to the best fitting sphere. The numbers below each map represent the average and standard deviation of the radius of curvature of best fitting sphere in $\mathrm{mm}$. 


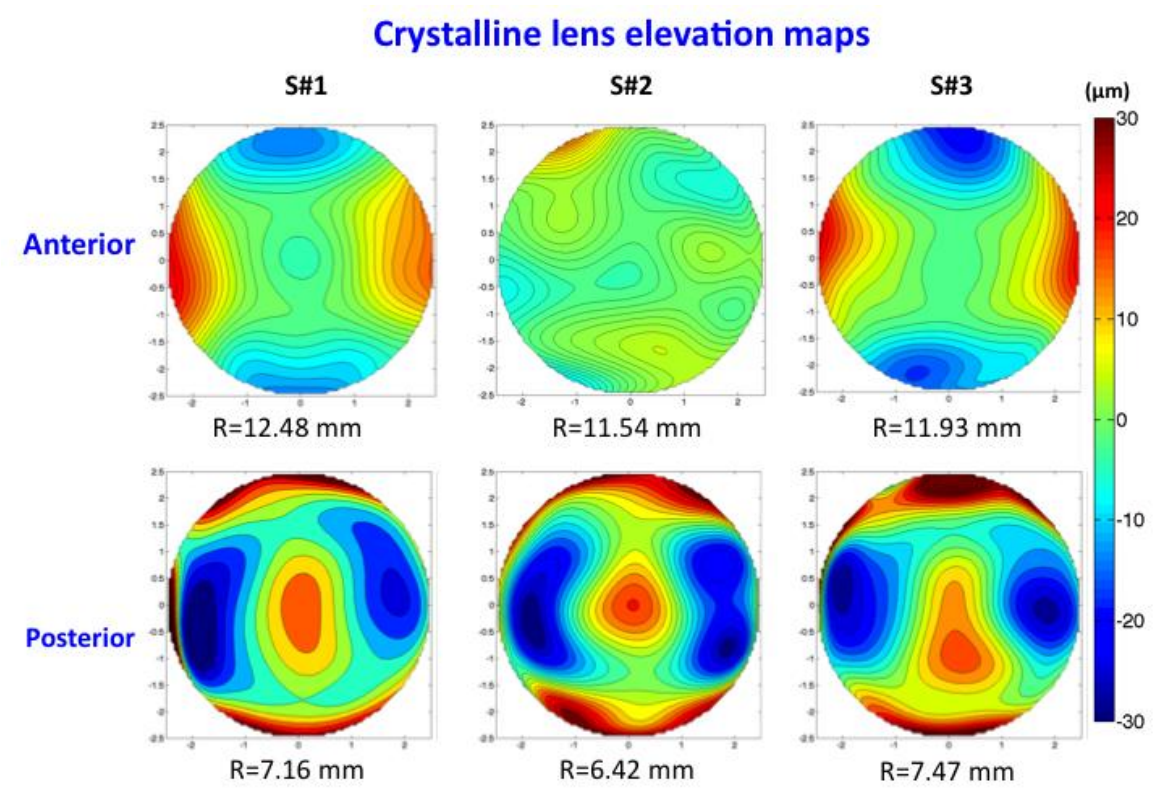

Fig 3. Quantitative anterior (top) and posterior (bottom) crystalline lens elevation maps in 3 eyes in vivo, after full distortion correction. Maps are Zernike fits to the elevation maps, relative to the best fitting sphere. $\mathrm{R}=$ radii of curvature of the best fitting sphere (from fits to sphere quadrics). Data are for 5-mm pupils.

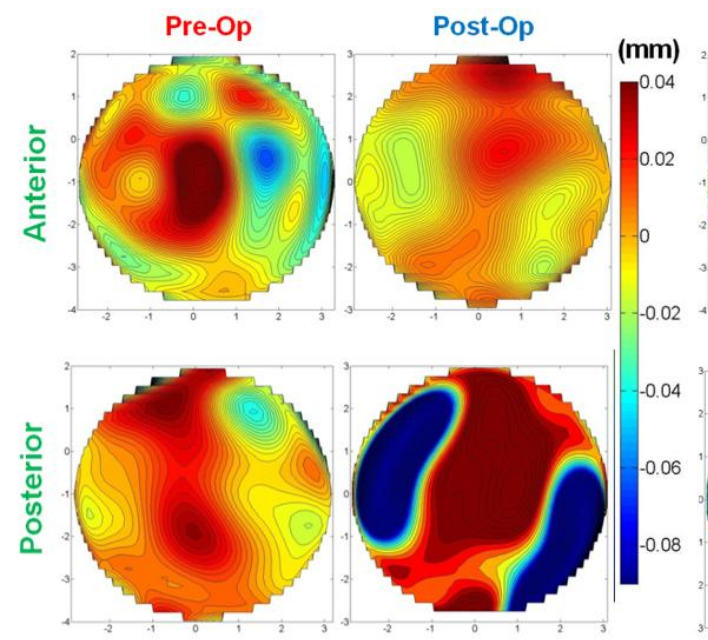

(a)

(b)

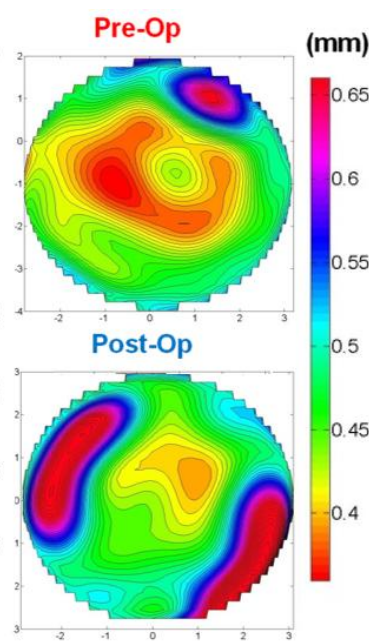

(c)

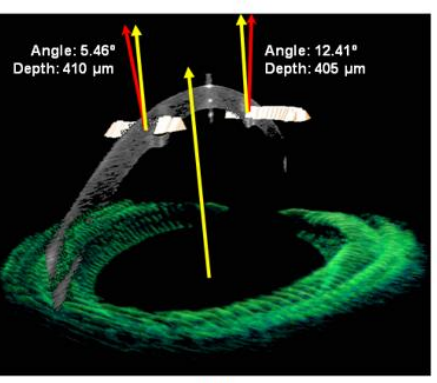

(d)

Fig. 4. (a) Quantitative anterior and posterior corneal elevation maps of a keratoconic cornea. (b) Quantitative post-ICRS anterior and posterior corneal elevation map. All data are after refraction distortion correction, and for a 6-mm zone.(c) Pachymetry map of keratoconic cornea (a) before (a) and (b) after ICRS implantation. Data are for a 6-mm zone. (d) 3-D analysis of the orientation (with respect to the pupil plane) and average depth (with respect to the anterior cornea) of the left and right ICRS. The red arrows are normal to the plane of the ICRS, and the yellow arrows are normal to the pupil.

\section{3.b. Lens topographic maps}

Figure 3 shows elevation maps of the anterior and posterior lens surfaces in the 3 subjects of the study, within a 5-mm diameter zone. The elevation maps represent 10th order Zernike fits of the elevation maps relative to the best fitting sphere. The numbers below each map indicate the radii of curvature of best fitting sphere.

\section{3.c. ICRS surgery characterization}

Figure 4 (a and b) shows quantitative topographies of the anterior and posterior corneal surfaces before and after ICRS implantation. The pre-operative anterior corneal surface shows a $40 \mu \mathrm{m}$ bulging (with respect to the reference sphere) near the ectactic zone, next to a $-45 \mu \mathrm{m}$ depression. Large asymmetries are also observed in the posterior cornea. After 
ICRS implantation, the anterior surface of the cornea shows a more regular pattern, a shift of the area of maximum elevation and a decrease in the maximum height (down to $15 \mu \mathrm{m}$.). The ICRS produces a stretching that decreases elevation $(-20 \mu \mathrm{m})$ in the area above the ICRS. The posterior topographic map is consistent with a lateral stretching in the optical zone induced by the action of ICRS, and shows an effective protrusion of the cornea areas behind the ICRS.

ICRS implantation increased the minimum corneal thickness (from $353 \pm 15 \mu \mathrm{m}$ preoperatively and $429 \pm 15 \mu \mathrm{m}$ post-ICRS), and decreased the overall corneal thickness asymmetry (Fig. 4(c)). The post-ICRS pachymetry map is consistent with a lateral stretching within the optical zone induced by the action of ICRS. At the location of the ICRS (i.e. beyond the optical zone) corneal thickness effectively increased, indicating an axial stretching of the cornea by the ICRS.

Figure 4(d) shows an illustration of the estimated location and orientation of the ICRS inside the cornea in 3-D. The ICRS center of mass was measured to be at a depth from the anterior corneal surface ranging from 350 to $470 \mu \mathrm{m}$ (left segment) and from 360 to 450 (right segment). The ICRS was tilted $5.46 \pm 1.16^{\circ}$ (left segment) and $12.41 \pm 1.65^{\circ}$ (right segment) with respect to the pupil plane, and laterally decentered $(-0.10 \pm 0.05 \mathrm{~mm}$ in the horizontal direction and

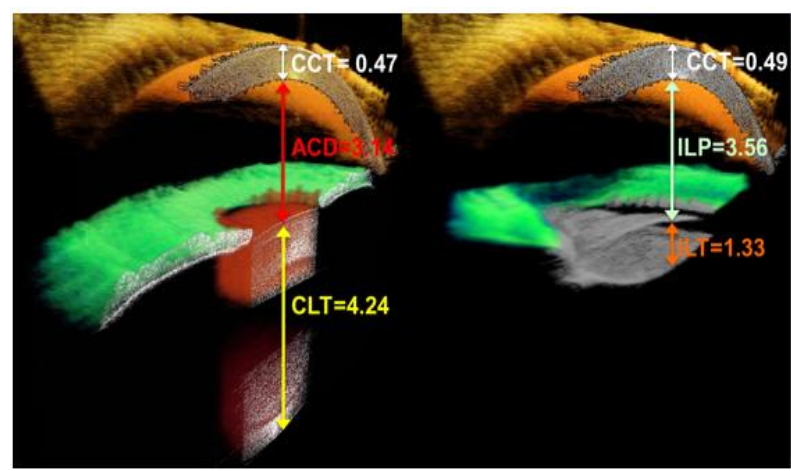

(a)

(b)
$-0.34 \pm 0.10 \mathrm{~mm}$ in the vertical direction) with respect to the pupil center.

\section{3.d. Biometry pre and post IOL implantation}

Figure 5 shows thickness and interocular distances of the ocular components (CCT, ACD/ILP and CLT/ILT), and tilt and decentration of the crystalline lens/IOL. The anterior surface of the IOL was placed $400 \mu \mathrm{m}$ behind the natural crystalline lens. CLT is consistent with the average 73-year old lens thickness [25]. Both the crystalline lens and IOL were tilted and decentered with respect to the pupillary axis in the same direction pre- and post- operatively, but their amounts changed significantly with surgery. According to existing conventions [24], negative horizontal decentration stands for nasal (in left eyes), and positive vertical decentration for superior. Positive tilt around the horizontal axis (Tilt $\mathrm{x}$ ) indicates that the superior edge of the lens is moved forward. Negative tilt around the vertical axis (Tilt y), in left eyes, indicates that the nasal edge of the lens is moved forward.

\section{Discussion}

This paper addresses the use of quantification techniques for characterizing the geometrical features of the anterior segment of the eye using Optical coherence tomography [14, 15 and 1720]. The developed methodology includes the development and calibration of OCTs for 3D imaging and geometrical characterization of the

Fig. 5. Illustration of the biometry evaluation from 3-D anterior segment AS-OCT and illustration of the lens tilt evaluation: Precataract surgery (a), and post-cataract surgery with IOL implantation. (a) Pre-cataract surgery: CCT: Central Corneal Thickness (in white), ACD: Anterior Chamber Depth (in red) and CLT: Central Lens Thickness (in yellow). (c) Post-cataract surgery with IOL implantation: CCT: Central Corneal Thickness (in white), ILP: Intraocular Lens Position (in light green) and ILT: Intraocular Lens Thickness (in orange). (d). Vector (in blue) is the pupillary axis, and (in purple) is the Lens/IOL axis. 
cornea and the lens, in normal, pathological and treated eyes.

Even though there are small statistically significant differences in terms of quadric parameters for the AS-OCT anterior corneal topographies without correction, it is in terms of the topographic maps where differences are most notorious. The presence of astigmatism in the data prior to fan distortion correction is clearly observed in the measurements of artificial surfaces, where (unlike in real eyes) no other astigmatism arising from alignment or shape should be present. Placido-based videokeratography, and to a less extent Scheimpflug imaging, are widely used clinically to measure the geometry of corneal surfaces. Although the radii of curvature of the surfaces were retrieved within $98.3 \%$ accuracy we did not attempt to explore the accuracy of the asphericity estimates.

We have presented accurate shape and 3-D topographies of the crystalline lens surfaces. We provided the first report of lens surface shapes in vivo from OCT upon correction of optical (refraction) distortion, although (as previous reports based on Purkinje or Scheimpflug) we did not considered a GRIN in the lens. Borja et al. [26] suggested that the optimal refractive index for the correction of OCT images was closer to the average refractive index [22] than to the equivalent refractive index. Our lens radii of curvature estimates in vivo $(11.90 \pm 1.34 \mathrm{~mm}$ for the anterior lens and $6.86 \pm 0.63 \mathrm{~mm}$ for the posterior lens) fall within the ranges reported in the literature. Classical eye model [27] used 10 $\mathrm{mm}$ for the anterior lens and $6 \mathrm{~mm}$ for the posterior lens, whereas more recent models [28] used 12.4 and $8.1 \mathrm{~mm}$ respectively. Dubbleman et al. [29] reported an age-dependent expression for the anterior and posterior lens radius of human lenses in vivo based on corrected Scheimpflug measurements, which applied to the average data of our three subjects would predict $11.02-11.3 \mathrm{~mm}$ and $5.8-5.86 \mathrm{~mm}$ for the anterior and posterior lens radius respectively.

We also presented tools for analysis of the keratoconic cornea. In this study, we provide extensive information of the geometrical features of the keratoconic corneas based on OCT imaging, including anterior and posterior corneal elevation maps, pachymetric maps, 3-D location of the ectatic area, corneal apex and pupil center for referencing This information is essential in planning treatment, in particular ICRS implantation.

Finally, we presented the followed methodology 3-D biometry of the anterior segment of the eye based on quantitative OCT, and its application in cataract surgery. 3-D measurements allowed interocular distances and thickness to be measured along the objectively identified axes (as opposed to single A-scan measurements which rely on patient's fixation). In addition, this is, to our knowledge, the first time that the crystalline lens and IOL tilt and decentration have been measured in 3-D using OCT. This information, along with full corneal topography [17-20] and axial length measurements, is extremely valuable to improve biometric pre-operative evaluations of cataract surgery, and therefore the selection of the most appropriate IOL design and power, as well as to fully characterize surgical outcomes and optical performance in pseudophakic eyes.

\section{Conclusions}

Anterior segment OCT provided with full distortion correction and automatic analysis tools allowed quantification of the human anterior segment of the eye in vivo and in 3-D. Fan distortion correction is essential to obtain reliable corneal elevation maps from AS-OCT in patients. The corrected topographies of the anterior cornea were comparable with topographies from state-of-the art clinical topography systems. The retrieved lens radii of curvature from OCT agree with phakometric data previously reported using Scheimpflug and Purkinke image along one meridian. We found slight negative lens surface asphericities, crossed astigmatism in the anterior and posterior lens surface, and low high order irregularities in the lens surfaces. Furthermore, OCT quantitative imaging allows comprehensive 3-D quantitative analysis of the keratoconic cornea and the changes produced by ICRS treatment, as well as monitoring of the ICRS three-dimensional location. ICRS produced a flattening of the anterior surface, steepening of 
the posterior surface, meridional differences in the changes of radii if curvature and asphericities, a symmetrization of the anterior corneal and a redistribution of corneal thickness. The biometric data pre and post-operative parameters CCT, ACD/ILP, CLT/ILT Tilt and decentration are retrieved with a very high degree of accuracy. IOL was placed $400 \mu \mathrm{m}$ behind the natural crystalline lens. In the reported patient the IOL tilt and decentration preserved the orientation of the natural lens (lens tilted with the superior and nasal edge forward, and decentered superiorly and nasally); pre-operative tilt was $3.11 \mathrm{deg}$ and decentration $0.77 \mathrm{~mm}$; post-operative tilt was $0.77 \mathrm{deg}$ and decentration $0.47 \mathrm{~mm}$. OCT-based biometry is a promising tool to investigate the changes after cataract surgery.

\section{Acknowledgements}

This study has been funded by Spanish Government Grant FIS2008-02065 and FIS201125637 and European Grants EURYI-05-102-ES (EURHORCs-ESF) and European Research Council Grant ERC-2011 AdG-294099 to S. Marcos. The authors acknowledge to all coauthors that participated in all these studies especially among them D. Siedlecki and P. Perez Merino. The authors also acknowledge the collaborative agreement "Unidad Asociada Tecnología e Innovación sanitaria en Oftalmología" (IIS Fundación Jiménez Díaz/ IOCSIC). 